\title{
A Study on Effects of Salinity on Growth and Yield of Tomato Genotype (Solanum lycopersicum)
}

\author{
Ja'afar Umar ${ }^{1, a^{*}}$, Adamu Aliyu², Kasimu Shehu², Lawal Abubakar ${ }^{3}$ \\ ${ }^{1}$ Department of Biology, Federal University Birnin Kebbi, Nigeria \\ ${ }^{2}$ Department of Biological Sciences, Usmanu Danfodiyo University, Sokoto, Nigeria \\ ${ }^{3}$ Department of Crop Science, Usmanu Danfodiyo University, Sokoto, Nigeria \\ *realumar2001@gmail.com
}

Keywords: Salinity, tomato, growth, yield.

\begin{abstract}
Saline water occupies $71 \%$ of the Earth area. It is thought that even a quarter of the whole pedosphere is affected by salts amounting to $950 \times 106$ ha while $23 \%$ of the $1.5 \times 109$ ha cultivated land is considered as saline. This study was carried out to investigate the influence of salinity on the on growth and yield of tomato genotypes. The seedlings 20 genotype were divided into three groups, Sodium chloride $(\mathrm{NaCl})$ dissolved in irrigation water to make variant concentration of 30 , $60 \mathrm{mg} / \mathrm{L}$ of salt concentration using E. C meter which were used to water the plants. Vegetative and reproductive characterization was evaluated. Data obtained were subjected to one way analysis of variance using SPSS (20) Statistical Software. All the genotypes exhibited a decline significantly in vegetative and reproductive parameters measured, with increasing salt concentration. In conclusion, the result of this research suggest that salinity decline both vegetative and reproductive parameters in tomato.
\end{abstract}

\section{Introduction}

Nigeria is the largest producer of tomato in Sub- Saharan Africa and $13^{\text {th }}$ in the world with an annual output of 2.4 million tones. Notwithstanding, the production faces challenges with distribution, storage, biotic and abiotic stress [5]. The decline in agricultural production in recent times in Nigeria (which declined from 7.2 percent in 2007 to 5.7 percent in 2011 [3]. This is not resultant of variations in rainfall but to other indicators of climate change such as increase in temperature and evaporation, drought and wind [21]. He also suggested that climate change measured by variation in rainfall may not directly affect agricultural production but indirectly through of the aftermaths of such rainfall such as erosion. Tomato plant suffers from various physiological problems in soil (abiotic stress) around the world. Until now, the causes behind such abnormalities in tomato cultivation have not been well understood. Wada et al. [20] reported that calcium concentration of young fruit decreased quickly when fruit fresh weight reaches an average of $20 \mathrm{~g}$. There is paucity of information on salt responsive candidate gene in tomato which could be useful in alleviating loss and low yield in tomato production.

Salinity is one of the major limitations on plant growth and productivity throughout the world. Damage caused to plants by high salinity is observed as either loss of plant productivity or plant death. Water sources on earth contain $30 \mathrm{~g}$ of sodium chloride per liter, making earth been referred to as a salty planet [4]. With the increase of soil salinity over the years due to poor irrigation and chemical application to the soil, it's expected that by 2050 , more than $50 \%$ of the land available for agriculture will be lost because of salinity [7]. Most tomato cultivars are sensitive to moderate levels of salinity [18]. Indeed, all plant development stages, including seed germination, vegetative growth and reproduction, show salinity sensitivity, that leads to poor harvests and reduced economic yield [23]. Tomato production has been limited due to high salinity level of the soil or of the irrigation water [22]. 


\section{Materials and Methods}

\section{Plant Materials}

The field experiment was conducted in the Biological garden of the Usmanu Danfodiyo University, Sokoto located at latitude $13^{0} 0^{\prime} 21.1428^{\prime \prime} \mathrm{N}$ and longitude $5^{\circ} 14^{\prime} 51.1872^{\prime \prime}$ E. Selected tomato landraces genotypes were obtained from local markets around Sokoto and Zamfara State Nigeia metropolis. These were identified in Heberium of Ahmadu Bello University, Zaria. While accessions genotypes seeds were obtained from Zamfara State Agricultural Development Project, Gusau (ZADP) and Institute for Agricultural Research (IAR) Ahmadu Bello University Zaria. The seeds of 20 genotypes of tomato were grouped into landraces and accessions, the collection location, type and common name of the cultivar are summarized on Table 1.

\section{Salinity Inducement}

The experiment was laid down in completely randomized design (CRD) consisting of three treatments (control with no concentration of Salt, 30 and $60 \mathrm{mgL}^{-1}$ of salt using E.C meter model, ORP 850081); the seedlings of each accession was divided into three groups: The first group represent the control where no salt was added. The second and third groups received 30 and $60 \mathrm{mgL}^{-1}$ of Salt respectively and replicated three times. The seeds were surface sterilized by soaking in 5\% sodium hypochlorite and rinsed three times in distilled water. The seeds were first sown in nursery beds and uniformly germinated seedlings ( 2 weeks old) were selected and transferred to poly bags containing a mixture of river sand and organic manure in 3:1 ratio.

Table 1. Different tomato genotypes used and their locations.

\begin{tabular}{|l|l|l|}
\hline Genotype & Type & Collection Locatity \\
\hline Dangainakawa & Landrace & Market \\
\hline Bahaushe & Landrace & Market \\
\hline Dandino & Landrace & Market \\
\hline Daneka & Landrace & Market \\
\hline Dan Gombe & Landrace & Market \\
\hline Dan Mazari & Landrace & Market \\
\hline Dandubukamiya & Landrace & Market \\
\hline Dankwandawa & Landrace & Market \\
\hline Ganwon falke & Landrace & Market \\
\hline Dan dogarawa & Landrace & Market \\
\hline Roma & Released & ZADP \\
\hline UTC & Released & ZADP \\
\hline Rio grande & Released & ZADP \\
\hline Giofranco F & Released & ZADP \\
\hline UC82B & Released & IARI \\
\hline Indian tomato & Released & IARI \\
\hline Tomato peto & Released & IARA \\
\hline Tropimech & Released & ZADP \\
\hline Cherry & Released & IARI \\
\hline Heirloom Tomato & Released & IARI \\
\hline
\end{tabular}

\section{Plant Height and Root Length}

After 28 days of salt treatment, salt tolerance was asses in both relative performance under salinity compared with control conditions for various yield parameters according to the method of Munns and James [13]. Plant height and root length parameters were assessed according to the International Plant Genetic Institute [9]. Plant height was measured from the base of the plant to the tip of the meristem, and root length was determined by measuring the length of the underground system using centimeter rule. 


\section{Number of Leaves, Floral Count and Number of Fruits}

The average number of leaves, number of flowers and number of fruits were determined by counting the entire number of leaves, flower and fruits divided by the number of plant in each pot [8].

$$
T N P \div N P=\text { Parameter } / \text { plant }
$$

where $\mathrm{TNP}=$ Total number of parameters and $\mathrm{NP}=$ Number of plant.

\section{Leaf area and Dry Matter Accumulation}

Leaf area was measured using Bohnert and Jensen [2] method by laying the leaves to be measured on a $1 \mathrm{~cm}$ grid and trace their outlines. The number of square centimeters the leaf covered was counted and estimated the area of the partial squares, at least half covered by the leaf [10]. Constant dry weight was determined after drying in an oven at $80^{\circ} \mathrm{C}$ to dry the entire moisture content and weighted. All the vegetative parameters were computed according to the Hunt [8] formula, to evaluate the physical level of salt tolerance in the selected cultivars.

\section{Fruit Size and Weight of Fruit at Harvest}

The size of the fruit was determined according to IPGRI descriptors of tomato chart [10]. The length was recorded from stem end to blossom end, to one decimal place at maturity. The fruit width was recorded at the largest diameter of cross-sectioned fruits to one decimal Place, at maturity. Fruits $<3 \mathrm{~cm}$ were described as very small while $3-5 \mathrm{~cm}$ small, $5.1-8 \mathrm{~cm}$ intermediate, 8 $10 \mathrm{~cm}$ large and $>10 \mathrm{~cm}$ were described as very large respectively. The weight of fruit of each plant at different treatments was determined by weighting the number of fruits in gram using weighing scale model CBB 600 [19].

\section{Fruit Shelf Life}

The shelf life of the fruits from different cultivars was determined by keeping the fruits at room temperature ( $35 \pm 2)$, for 10 day after harvesting, between 5-8 days is considered to have long shelf life, 3- 5 days medium shelf life and 0- 3 days short shelf life [10].

\section{Results}

\section{Phenotypic and Biochemical Responses of Tomato to Salinity}

Salinity significantly reduced plant vegetative parameters (number of leaves, plant height, root length, leaves area and Dry Matter Accumulation) in concentration dependent manner in all the twenty genotype. The highest number of leaves were recorded in control of Riogrande and Daneka with 88.67 leaves per plant each and the lowest leaves count were recorded in Dankwandawa and Dan gainakawa treated with $60 \mathrm{mgL}-1$ of salt with 16.67 and 17.00 leaves per plant (Table 2). However, Plant height significantly $(\mathrm{P}<0.05)$ affected by salt concentration in all the genotype used. Daneka recorded the highest plant height of 61.00 at control and Dandubukamiya recorded the highest plant height in plant treated with $60 \mathrm{mgL}-1$ of salt with $38.67 \mathrm{~cm}$ (Table 2).

Root length significantly affected by salinity in concentration dependant manner. However, the lowest length of root at control of $3.03 \mathrm{~cm}$ was recorded in Dankwandawa followed by Ganwon falke with $3.70 \mathrm{~cm}$. The highest root length in plants treated with $30 \mathrm{mgL}-1$ of Salinity was recorded in Daneka with $10.17 \mathrm{~cm}$ and the lowest root length in plant treated with $30 \mathrm{mgL}-1$ of Salinity was observed in UTC and Dandogarawa with $2.27 \mathrm{~cm}$ each (Table 2). Leaf area also significantly $(\mathrm{P}<0.05)$ affected by salinity. The largest leave area of $27.30 \mathrm{sq} / \mathrm{m}$ was recorded in Tropimech followed by Roma with $24.67 \mathrm{sq} / \mathrm{m}$ (Table 2). Dry Matter Accumulation significantly affected by salinity episode in all the genotypes. The lowest Dry Matter Accumulation of $0.90 \mathrm{~g}$ was recorded in Dangainakawa treated with $60 \mathrm{mgL}-1$ of Salinity followed by Dandogarawa with $1.60 \mathrm{~g}$ respectively (Table 2). Number of flowers, number of fruits and fruit weight at harvest were significantly 
$(\mathrm{P}<0.05)$ affected by salinity in concentration dependent manner. Highest number of flowers (35/plant) was recorded at control in Dan Mazari followed by Indian tomato (released cultivar) with 33 flowers per plant (Table 3). The lowest number of flowers was recorded on plant treated with $60 \mathrm{mgL}-1$ of Salinity on Dan Gainakawa with 5.67 flowers per plant followed by Dan Eka with 6.67 flowers (Table 3).

Highest number of fruit was recorded at control on Indian tomato with 28 fruits followed by Tomato Peto with 26 fruits. The lowest number of fruits was recorded at plant treated with $60 \mathrm{mgL}-$ 1 of Salinity on Dan Gombe with 8 fruits followed by Dandino with 10 fruits (Table 3). Fruit weight at harvest also significantly $(\mathrm{P}<0.05)$ affected by salinity. Highest fruit weight was observed in control among the twenty accessions and decreased as the salt concentration increased. The highest fruit weight of $136.03 \mathrm{~g} /$ fruit was recorded in control in Dan Dogarawa followed by Dan Gombe with $107.50 \mathrm{~g}$. The lowest fruit weight of $15.00 \mathrm{~g} /$ fruit was recorded in Dan Gainakawa. However, UC 82B and UTC recorded the same fruit weight in control with $104.30 \mathrm{~g}$ each respectively (Table 3 ).

Table 2: Effects of Salt Concentration on Vegetative Characters in Different Tomato Genotypes.

\begin{tabular}{|c|c|c|c|c|c|c|}
\hline $\begin{array}{l}\text { Accessions/ } \\
\text { Landraces }\end{array}$ & $\begin{array}{c}\text { Treatment } \\
\text { (mgL-1) }\end{array}$ & $\begin{array}{c}\text { Number of } \\
\text { leaves }\end{array}$ & Plant Height & $\begin{array}{c}\text { Root } \\
\text { Length }\end{array}$ & $\begin{array}{c}\text { Leaf Area } \\
(\mathrm{Sq} / \mathrm{m})\end{array}$ & $\begin{array}{c}\text { Dry Mass } \\
\text { Accumulation } \\
\text { (g) }\end{array}$ \\
\hline \multirow{4}{*}{ D/ Gainakaw } & 0 & $79.00^{\mathrm{a}} \pm 21.78$ & $45.67^{\mathrm{a}} \pm 2.50$ & $13.67^{\mathrm{a}} \pm 5.51$ & $6.30^{\mathrm{a}} \pm 0.57$ & $4.60^{\mathrm{a}} \pm 0.66$ \\
\hline & 30 & $45.00^{\mathrm{b}} \pm 12.10$ & $40.67^{b} \pm 1.16$ & $7.67^{\mathrm{b}} \pm 0.58$ & $5.30^{\mathrm{b}} \pm 0.57$ & $1.97^{\mathrm{b}} \pm 0.58$ \\
\hline & 60 & $17.00^{\mathrm{c}} \pm 15.13$ & $9.18^{c} \pm 10.00$ & $4.50^{c} \pm 3.90$ & $2.67^{\mathrm{c}} \pm 2.52$ & $0.90^{\mathrm{c}} \pm 0.80$ \\
\hline & LSD & 4.45 & 1.30 & 0.80 & 0.56 & 0.45 \\
\hline \multirow{4}{*}{ Bahaushe } & 0 & $74.30^{\mathrm{a}} \pm 6.10$ & $40.30^{\mathrm{a}} \pm 5.63$ & $12.80^{\mathrm{a}} \pm 4.50$ & $10.30^{\mathrm{a}} \pm 3.20$ & $4.40^{\mathrm{a}} \pm 0.46$ \\
\hline & 30 & $48.67^{\mathrm{b}} \pm 3.78$ & $28.00^{\mathrm{b}} \pm 5.59$ & $7.47^{\mathrm{b}} \pm 1.27$ & $8.67^{b} \pm 0.58$ & $3.67^{\mathrm{b}} \pm 0.12$ \\
\hline & 60 & $33.67^{\mathrm{c}} \pm 4.64$ & $24.30^{c} \pm 3.79$ & $3.97^{\mathrm{c}} \pm 0.50$ & $7.30^{c} \pm 0.58$ & $1.87^{\mathrm{c}} \pm 0.15$ \\
\hline & LSD & 4.35 & 2.05 & 1.00 & 0.95 & 0.65 \\
\hline \multirow{3}{*}{ Dandino } & 0 & $79.30^{\mathrm{a}} \pm 13.60$ & $54.20^{\mathrm{a}} \pm 6.42$ & $12.80^{\mathrm{a}} \pm 4.50$ & $20.67^{\mathrm{a}} \pm 4.04$ & $5.70^{\mathrm{a}} \pm 1.60$ \\
\hline & 30 & $58.30^{\mathrm{b}} \pm 7.64$ & $40.90^{\mathrm{b}} \pm 4.40$ & $7.47^{\mathrm{b}} \pm 1.27$ & $8.67^{\mathrm{b}} \pm 2.08$ & $4.87^{\mathrm{b}} \pm 0.15$ \\
\hline & 60 & $43.00^{c} \pm 2.00$ & $33.40^{\mathrm{c}} \pm 2.98$ & $4.00^{c} \pm 0.56$ & $4.67^{\mathrm{c}} \pm 1.53$ & $4.00^{\mathrm{c}} \pm 0.10$ \\
\hline \multirow{5}{*}{ Dan Eka } & LSD & $\mathbf{5 . 0 7}$ & 2.00 & 1.03 & 0.76 & 0.50 \\
\hline & 0 & $88.67^{\mathrm{a}} \pm 3.20$ & $61.00^{\mathrm{a}} \pm 5.57$ & $14.67^{\mathrm{a}} \pm 4.07$ & $18.67^{\mathrm{a}} \pm 2.31$ & $5.93^{\mathrm{a}} \pm 0.40$ \\
\hline & 30 & $62.67^{b} \pm 24.83$ & $48.07^{\mathrm{b}} \pm 7.20$ & $10.17^{\mathrm{b}} \pm 2.78$ & $15.30^{\mathrm{b}} \pm 0.58$ & $4.30^{\mathrm{b}} \pm 1.94$ \\
\hline & 60 & $34.67^{\mathrm{c}} \pm 13.70$ & $30.30^{c} \pm 8.90$ & $6.60^{\mathrm{c}} \pm 2.90$ & $13.00^{c} \pm 1.00$ & $2.00^{\mathrm{c}} \pm 0.30$ \\
\hline & LSD & 4.90 & 1.65 & 1.07 & 1.03 & 0.65 \\
\hline \multirow{3}{*}{ Dan Gombe } & 0 & $58.30^{\mathrm{a}} \pm 6.66$ & $43.47^{\mathrm{a}} \pm 5.62$ & $13.60^{\mathrm{a}} \pm 2.14$ & $21.30^{\mathrm{a}} \pm 2.30$ & $3.67^{\mathrm{a}} \pm 0.70$ \\
\hline & 30 & $39.67^{b} \pm 9.50$ & $33.07^{\mathrm{b}} \pm 2.90$ & $7.80^{\mathrm{b}} \pm 3.90$ & $16.30^{\mathrm{b}} \pm 0.58$ & $2.07^{\mathrm{b}} \pm 0.21$ \\
\hline & 60 & $24.00^{c} \pm 12.53$ & $20.17^{\mathrm{c}} \pm 2.63$ & $3.50^{\mathrm{c}} \pm 0.48$ & $14.30^{c} \pm 2.08$ & $1.47^{\mathrm{c}} \pm 0.30$ \\
\hline \multirow{4}{*}{ Dan Mazari } & LSD & 3.68 & 2.03 & 1.00 & 1.04 & 0.39 \\
\hline & 0 & $33.30^{\mathrm{a}} \pm 4.10$ & $26.67^{\mathrm{a}} \pm 1.53$ & $5.90^{\mathrm{a}} \pm 0.80$ & $16.30^{\mathrm{a}} \pm 0.58$ & $4.20^{\mathrm{a}} \pm 0.21$ \\
\hline & 30 & $28.00^{\mathrm{b}} \pm 1.00$ & $20.50^{\mathrm{b}} \pm 2.10$ & $4.50^{\mathrm{b}} \pm 0.36$ & $14.67^{b} \pm 0.58$ & $3.10^{\mathrm{b}} \pm 0.10$ \\
\hline & 60 & $23.67^{\mathrm{b}} \pm 0.57$ & $18.00^{\mathrm{b}} \pm 0.30$ & $3.50^{\mathrm{c}} \pm 0.26$ & $12.00^{c} \pm 1.00$ & $1.87^{\mathrm{c}} \pm 0.50$ \\
\hline \multirow{4}{*}{ Dan Dubu } & LSD & 4.53 & 2.90 & 0.89 & 0.90 & 0.40 \\
\hline & 0 & $55.30^{\mathrm{a}} \pm 2.08$ & $48.67^{\mathrm{a}} \pm 1.53$ & $6.77^{\mathrm{a}} \pm 0.21$ & $18.67^{\mathrm{a}} \pm 6.58$ & $5.10^{\mathrm{a}} \pm 0.10$ \\
\hline & 30 & $50.00^{\mathrm{b}} \pm 1.00$ & $44.00^{\mathrm{a}} \pm 1.00$ & $3.80^{\mathrm{b}} \pm 1.32$ & $15.00^{\mathrm{b}} \pm 2.00$ & $4.00^{\mathrm{b}} \pm 0.06$ \\
\hline & 60 & $47.67^{\mathrm{b}} \pm 0.58$ & $38.67^{\mathrm{b}} \pm 2.52$ & $2.60^{\mathrm{b}} \pm 1.40$ & $13.67^{\mathrm{b}} \pm 1.53$ & $3.27^{\mathrm{c}} \pm 0.06$ \\
\hline \multirow{4}{*}{ D/ Kwandawa } & LSD & 3.00 & 4.48 & 1.21 & 2.90 & 0.54 \\
\hline & 0 & $23.30^{\mathrm{a}} \pm 1.53$ & $19.67^{\mathrm{a}} \pm 4.73$ & $3.03^{\mathrm{a}} \pm 0.06$ & $7.10^{\mathrm{a}} \pm 1.88$ & $3.67^{\mathrm{a}} \pm 1.53$ \\
\hline & 30 & $19.67^{\mathrm{b}} \pm 4.04$ & $19.67^{\mathrm{b}} \pm 1.53$ & $2.40^{\mathrm{a}} \pm 0.04$ & $2.07^{\mathrm{b}} \pm 0.21$ & $2.00^{\mathrm{b}} \pm 1.70$ \\
\hline & 60 & $16.67^{\mathrm{b}} \pm 1.55$ & $10.67^{\mathrm{c}} \pm 0.58$ & $1.67^{\mathrm{a}} \pm 0.10$ & $1.90^{c} \pm 0.93$ & $1.67^{\mathrm{b}} \pm 1.10$ \\
\hline \multirow{4}{*}{ Ganwon Falke } & LSD & 3.04 & 0.90 & 1.50 & 2.01 & 1.00 \\
\hline & 0 & $33.67^{\mathrm{a}} \pm 4.73$ & $20.30^{\mathrm{a}} \pm 2.52$ & $3.70^{\mathrm{a}} \pm 0.67$ & $23.00^{\mathrm{a}} \pm 2.65$ & $4.17^{\mathrm{a}} \pm 0.15$ \\
\hline & 30 & $19.30^{\mathrm{b}} \pm 2.08$ & $17.00^{\mathrm{b}} \pm 1.70$ & $1.37^{\mathrm{b}} \pm 0.12$ & $17.30^{\mathrm{b}} \pm 0.58$ & $3.20^{\mathrm{a}} \pm 0.26$ \\
\hline & 60 & $16.30^{\mathrm{b}} \pm 058$ & $10.30^{c} \pm 1.50$ & $0.97^{\mathrm{b}} \pm 0.10$ & $14.30^{\mathrm{b}} \pm 0.58$ & $2.00^{\mathrm{a}} \pm 0.10$ \\
\hline \multirow{5}{*}{ D. Dogarawa } & LSD & 5.89 & 2.78 & 1.98 & 3.97 & 2.86 \\
\hline & 0 & $32.30^{\mathrm{a}} \pm 1.10$ & $23.77^{\mathrm{a}} \pm 3.30$ & $2.77^{\mathrm{a}} \pm 0.99$ & $12.30^{\mathrm{a}} \pm 1.50$ & $3.60^{\mathrm{a}} \pm 0.78$ \\
\hline & 30 & $23.00^{\mathrm{b}} \pm 2.00$ & $18.57^{\mathrm{b}} \pm 1.60$ & $2.27^{\mathrm{a}} \pm 0.76$ & $8.67^{b} \pm 0.58$ & $2.70^{\mathrm{b}} \pm 0.38$ \\
\hline & 60 & $20.00^{c} \pm 3.60$ & $15.20^{c} \pm 0.71$ & $0.97^{\mathrm{b}} \pm 1.50$ & $7.00^{\mathrm{b}} \pm 1.00$ & $1.60^{c} \pm 0.30$ \\
\hline & LSD & 2.09 & 1.09 & 0.98 & 1.97 & 0.87 \\
\hline
\end{tabular}


Table 2 Continued: Effects of Salt Concentration on Vegetative Characters in Different Tomato Cultivars.

\begin{tabular}{|c|c|c|c|c|c|c|}
\hline $\begin{array}{l}\text { Accessions/ } \\
\text { Landraces }\end{array}$ & $\begin{array}{c}\text { Treatment } \\
\text { (mgL-1) }\end{array}$ & $\begin{array}{l}\text { Number of } \\
\text { leaves }\end{array}$ & Plant Height & Root Length & $\begin{array}{l}\text { Leaf Area } \\
\text { (Sq/m) }\end{array}$ & $\begin{array}{c}\text { Dry Mass } \\
\text { Accumulation } \\
\text { (g) }\end{array}$ \\
\hline \multirow{4}{*}{ Roma } & 0 & $57.00^{\mathrm{a}} \pm 6.08$ & $46.40^{\mathrm{a}} \pm 5.30$ & $27.10^{\mathrm{a}} \pm 3.80$ & $24.67^{\mathrm{a}} \pm 1.53$ & $5.40^{\mathrm{a}} \pm 0.80$ \\
\hline & 30 & $40.00^{\mathrm{b}} \pm 5.00$ & $40.00^{\mathrm{b}} \pm 5.70$ & $3.87^{\mathrm{b}} \pm 0.98$ & $15.67^{\mathrm{b}} \pm 3.20$ & $4.00^{\mathrm{a}} \pm 0.20$ \\
\hline & 60 & $26.30^{c} \pm 5.50$ & $20.30^{c} \pm 5.50$ & $1.77^{\mathrm{c}} \pm 0.20$ & $11.67^{\mathrm{c}} \pm 2.51$ & $2.80^{\mathrm{a}} \pm 0.29$ \\
\hline & LSD & 3.87 & 1.09 & 1.98 & 0.43 & 2.08 \\
\hline \multirow{4}{*}{ UTC } & 0 & $69.67 \mathrm{a} \pm 1.53$ & $45.90 \mathrm{a} \pm 5.58$ & $9.00 \mathrm{a} \pm 2.00$ & $13.00 \mathrm{a} \pm 1.70$ & $6.00 \mathrm{a} \pm 0.10$ \\
\hline & 30 & $56.00 \mathrm{a} \pm 14.18$ & $33.00 \mathrm{~b} \pm 3.47$ & $4.67 \mathrm{~b} \pm 1.26$ & $9.00 \mathrm{~b} \pm 1.00$ & $5.10 \mathrm{a} \pm 0.70$ \\
\hline & 60 & $47.30 \mathrm{a} \pm 10.02$ & $31.00 \mathrm{~b} \pm 10.02$ & $2.80 \mathrm{c} \pm 1.10$ & $7.30 \mathrm{c} \pm 0.58$ & $3.40 \mathrm{~b} \pm 0.64$ \\
\hline & LSD & 14.87 & 3.42 & 0.54 & 0.76 & 1.53 \\
\hline \multirow{3}{*}{ Rio Grande } & 0 & $88.67 \mathrm{a} \pm 3.21$ & $48.10 \mathrm{a} \pm 2.70$ & $5.50 \mathrm{a} \pm 0.90$ & $20.00 \mathrm{a} \pm 3.60$ & $6.70 \pm 0.11$ \\
\hline & 30 & $70.30 \mathrm{~b} \pm 4.50$ & $33.77 \mathrm{~b} \pm 4.00$ & $3.10 \mathrm{~b} \pm 0.30$ & $15.30 \mathrm{~b} \pm 0.58$ & $5.60 \pm 0.46$ \\
\hline & 60 & $51.30 \mathrm{c} \pm 11.00$ & $25.07 \mathrm{c} \pm 2.83$ & $2.50 \mathrm{~b} \pm 0.50$ & $13.00 \mathrm{c} \pm 1.00$ & $4.70 \pm 0.70$ \\
\hline \multirow{5}{*}{ Gianfranco F. } & LSD & 5.12 & 2.07 & 0.76 & 0.75 & 0.87 \\
\hline & 0 & $79.00 \mathrm{a} \pm 16.40$ & $47.27 \mathrm{a} \pm 2.64$ & $5.07 \mathrm{a} \pm 0.93$ & $14.67 \mathrm{a} \pm 0.58$ & $5.97 \mathrm{a} \pm 0.29$ \\
\hline & 30 & $56.67 \mathrm{~b} \pm 10.40$ & $33.90 \mathrm{~b} \pm 5.40$ & $3.30 \mathrm{~b} \pm 0.26$ & $12.67 \mathrm{a} \pm 0.58$ & $4.70 \mathrm{~b} \pm 0.70$ \\
\hline & 60 & $33.67 \mathrm{c} \pm 5.51$ & $25.50 \mathrm{c} \pm 4.76$ & $2.80 \mathrm{~b} \pm 0.26$ & $8.67 \mathrm{~b} \pm 1.10$ & $2.89 \mathrm{c} \pm 0.39$ \\
\hline & LSD & 5.70 & 2.97 & 1.31 & 2.53 & 1.02 \\
\hline \multirow{3}{*}{ UC82B } & 0 & $70.30 \mathrm{a} \pm 13.00$ & $44.47 \mathrm{a} \pm 5.06$ & $11.40 \mathrm{a} \pm 14.32$ & $16.67 \mathrm{a} \pm 0.57$ & $5.40 \mathrm{a} \pm 0.60$ \\
\hline & 30 & $58.00 \mathrm{~b} \pm 2.60$ & $29.00 \mathrm{~b} \pm 1.70$ & $2.27 \mathrm{~b} \pm 0.25$ & $14.30 \mathrm{~b} \pm 1.10$ & $4.50 \mathrm{ab} \pm 0.40$ \\
\hline & 60 & $44.30 \mathrm{c} \pm 5.10$ & $23.97 \mathrm{c} \pm 4.38$ & $2.30 \mathrm{~b} \pm 0.60$ & $12.30 \mathrm{~b} \pm 0.57$ & $2.80 \mathrm{~b} \pm 0.76$ \\
\hline \multirow{5}{*}{ Indian tomato } & LSD & 6.98 & 3.86 & 2.06 & 2.00 & 1.96 \\
\hline & 0 & $62.30 \mathrm{a} \pm 5.86$ & $41.30 \mathrm{a} \pm 1.50$ & $7.77 \mathrm{a} \pm 3.00$ & $9.67 \mathrm{a} \pm 0.57$ & $5.00 \mathrm{a} \pm 0.58$ \\
\hline & 30 & $53.30 \mathrm{~b} \pm 4.90$ & $34.30 \mathrm{~b} \pm 4.00$ & $8.00 \mathrm{a} \pm 0.60$ & $9.00 \mathrm{~b} \pm 0.00$ & $4.37 \mathrm{~b} \pm 0.40$ \\
\hline & 60 & $46.67 \mathrm{~b} \pm 2.89$ & $33.67 \mathrm{~b} \pm 4.00$ & $5.07 \mathrm{~b} \pm 1.08$ & $7.67 \mathrm{c} \pm 50.90$ & $3.10 \mathrm{c} \pm 0.10$ \\
\hline & LSD & 6.87 & 1.98 & 0.94 & 0.56 & 0.23 \\
\hline \multirow{3}{*}{ Tomato Peto } & 0 & $59.00 \mathrm{a} \pm 5.29$ & $41.30 \mathrm{a} \pm 1.50$ & $6.00 \mathrm{a} \pm 0.80$ & $21.30 \mathrm{a} \pm 1.50$ & $5.27 \mathrm{a} \pm 0.10$ \\
\hline & 30 & $47.67 \mathrm{~b} \pm 9.40$ & $35.00 \mathrm{~b} \pm 4.00$ & $4.70 b \pm 0.38$ & $18.67 \mathrm{~b} \pm 1.10$ & $4.17 b \pm 0.20$ \\
\hline & 60 & $43.67 \mathrm{~b} \pm 8.08$ & $29.67 \mathrm{c} \pm 0.58$ & $4.10 c \pm 1.50$ & $14.30 \mathrm{c} \pm 1.10$ & $2.00 \mathrm{c} \pm 0.80$ \\
\hline \multirow{5}{*}{ Tropimech } & LSD & 4.67 & 1.04 & 0.45 & 0.64 & 0.50 \\
\hline & 0 & $69.30 \mathrm{a} \pm 5.51$ & $38.30 \mathrm{a} \pm 1.00$ & $4.00 \mathrm{a} \pm 0.71$ & $27.30 \mathrm{a} \pm 2.08$ & $6.00 \mathrm{a} \pm 0.56$ \\
\hline & 30 & $64.00 \mathrm{~b} \pm 2.61$ & $33.00 \mathrm{~b} \pm 2.83$ & $3.17 \mathrm{ab} \pm 0.10$ & $21.00 \mathrm{~b} \pm 4.00$ & $5.70 \mathrm{a} \pm 0.61$ \\
\hline & 60 & $53.30 \mathrm{c} \pm 2.52$ & $21.30 \mathrm{c} \pm 1.50$ & $2.80 \mathrm{~b} \pm 0.10$ & $16.00 \mathrm{c} \pm 1.70$ & $4.40 \mathrm{~b} \pm 0.66$ \\
\hline & LSD & 3.23 & 2.43 & 0.98 & 1.00 & 0.76 \\
\hline \multirow{3}{*}{ Cherry } & 0 & $33.67 \mathrm{a} \pm 3.20$ & $28.87 \mathrm{a} \pm 3.59$ & $7.87 \mathrm{a} \pm 2.20$ & $15.30 \mathrm{a} \pm 0.58$ & $4.17 \mathrm{a} \pm 0.12$ \\
\hline & 30 & $24.30 \mathrm{~b} \pm 3.79$ & $25.17 \mathrm{~b} \pm 0.90$ & $4.07 \mathrm{~b} \pm 2.50$ & $13.30 \mathrm{~b} \pm 1.50$ & $3.10 \mathrm{~b} \pm 0.10$ \\
\hline & 60 & $21.00 \mathrm{c} \pm 2.00$ & $19.43 c \pm 3.60$ & $1.97 \mathrm{c} \pm 0.35$ & $12.67 \mathrm{~b} \pm 1.50$ & $2.00 \mathrm{c} \pm 0.10$ \\
\hline \multirow{5}{*}{ Heirloom } & LSD & 1.67 & 1.54 & 0.34 & 0.94 & 0.45 \\
\hline & 0 & $66.67 a \pm 3.50$ & $43.23 \mathrm{a} \pm 2.90$ & $5.70 \mathrm{a} \pm 0.80$ & $16.67 \mathrm{a} \pm 2.08$ & $5.30 \mathrm{a} \pm 0.30$ \\
\hline & 30 & $55.00 \mathrm{~b} \pm 2.60$ & $37.87 \mathrm{~b} \pm 1.30$ & $4.17 \mathrm{~b} \pm 0.50$ & $9.00 \mathrm{~b} \pm 1.70$ & $4.30 \mathrm{~b} \pm 0.43$ \\
\hline & 60 & $46.00 \mathrm{c} \pm 4.00$ & $31.07 \mathrm{c} \pm 1.96$ & $3.20 \mathrm{c} \pm 0.20$ & $7.00 \mathrm{c} \pm 0.00$ & $3.00 \mathrm{c} \pm 0.10$ \\
\hline & LSD & 4.45 & 2.76 & 0.74 & 0.89 & 0.54 \\
\hline
\end{tabular}

Values represent means and standard deviation of vegetative parameters. Mean in a column with the same superscript are not significantly different at $(\mathrm{P}<0.05)$ 
Table 3: Effects of salt concentration on floral characters, number of fruits and fruits weight at harvest in different tomato genotypes studied.

\begin{tabular}{|c|c|c|c|c|}
\hline $\begin{array}{l}\text { Accessions/ } \\
\text { Landraces }\end{array}$ & Treatment & Number of Flowers & Number of Fruit & Fruit Weight (g) \\
\hline \multirow[t]{4}{*}{ D. Gainakawa } & 0 & $28.00^{\mathrm{a}} \pm 7.55$ & $12.00^{\mathrm{a}} \pm 2.65$ & $15.03^{\mathrm{a}} \pm 0.58$ \\
\hline & 30 & $16.67^{\mathrm{b}} \pm 4.90$ & $10.00^{\mathrm{a}} \pm 2.65$ & $14.10^{\mathrm{b}} \pm 0.58$ \\
\hline & 60 & $5.67^{c} \pm 5.10$ & $4.00 \pm^{\mathrm{b}} 4.00$ & $9.27^{\mathrm{c}} \pm 8.01$ \\
\hline & LSD & 2.67 & 2.03 & 1.06 \\
\hline \multirow[t]{4}{*}{ Bahaushe } & 0 & $27.00^{\mathrm{a}} \pm 6.08$ & $13.67^{\mathrm{a}} \pm 3.79$ & $157.70^{\mathrm{a}} \pm 2.89$ \\
\hline & 30 & $21.30^{b} \pm 5.69$ & $9.30 \pm^{\mathrm{b}} 0.58$ & $92.70^{b} \pm 1.28$ \\
\hline & 60 & $7.00 \pm^{\mathrm{c}} 1.00$ & $3.67 \pm{ }^{\mathrm{c}} 0.58$ & $65.70^{c} \pm 3.00$ \\
\hline & LSD & 2.89 & 1.02 & 8.90 \\
\hline \multirow[t]{4}{*}{ Dandino } & 0 & $17.00^{\mathrm{a}} \pm 2.00$ & $10.00^{\mathrm{a}} \pm 1.00$ & $100.1^{\mathrm{a}} 0 \pm 1.60$ \\
\hline & 30 & $14.67^{\mathrm{b}} \pm 0.58$ & $8.67^{\mathrm{b}} \pm 2.00$ & $97.40^{\mathrm{a}} \pm 0.50$ \\
\hline & 60 & $9.00^{c} \pm 1.00$ & $4.67^{\mathrm{c}} \pm 1.53$ & $74.90 \mathrm{~b} \pm 1.60$ \\
\hline & LSD & 2.01 & 1.00 & 6.87 \\
\hline \multirow[t]{4}{*}{ Dan Eka } & 0 & $21.00^{\mathrm{a}} \pm 1.70$ & $14.00^{\mathrm{a}} \pm 1.00$ & $59.80^{\mathrm{a}} \pm 3.50$ \\
\hline & 30 & $13.00^{\mathrm{b}} \pm 5.20$ & $8.00^{\mathrm{b}} \pm 5.10$ & $45.70^{\mathrm{b}} \pm 3.17$ \\
\hline & 60 & $6.67^{\mathrm{c}} \pm 1.53$ & $3.67^{\mathrm{c}} \pm 1.10$ & $30.70^{c} \pm 3.12$ \\
\hline & LSD & 2.00 & 1.05 & 3.67 \\
\hline \multirow[t]{4}{*}{ Dan Gombe } & 0 & $12.67^{\mathrm{a}} \pm 3.79$ & $8.30^{\mathrm{a}} \pm 3.20$ & $107.50^{\mathrm{a}} \pm 1.70$ \\
\hline & 30 & $9.30^{\mathrm{b}} \pm 0.58$ & $5.00^{\mathrm{b}} \pm 1.00$ & $64.10^{\mathrm{b}} \pm 1.15$ \\
\hline & 60 & $6.67^{\mathrm{c}} \pm 0.58$ & $3.30^{\mathrm{c}} \pm 1.10$ & $60.87^{\mathrm{b}} \pm 0.67$ \\
\hline & LSD & 1.04 & 0.90 & 4.89 \\
\hline \multirow[t]{4}{*}{ Dan Mazari } & 0 & $35.30^{\mathrm{a}} \pm 5.50$ & $21.00^{\mathrm{a}} \pm 2.00$ & $67.20^{\mathrm{a}} \pm 7.70$ \\
\hline & 30 & $26.67^{\mathrm{b}} \pm 4.04$ & $14.67^{\mathrm{b}} \pm 1.53$ & $58.60^{\mathrm{b}} \pm 1.40$ \\
\hline & 60 & $18.00^{c} \pm 2.65$ & $8.30^{c} \pm 1.53$ & $43.90^{c} \pm 0.50$ \\
\hline & LSD & 2.89 & 1.50 & 4.02 \\
\hline \multirow[t]{4}{*}{ Dan Dubu } & 0 & $5.10^{\mathrm{a}} \pm 0.10$ & $21.00^{\mathrm{a}} \pm 6.56$ & $44.30^{\mathrm{a}} \pm 1.10$ \\
\hline & 30 & $4.00^{\mathrm{b}} \pm 0.58$ & $16.30^{\mathrm{b}} \pm 2.30$ & $34.30^{\mathrm{b}} \pm 0.58$ \\
\hline & 60 & $3.37^{\mathrm{c}} \pm 0.58$ & $9.67^{c} \pm 3.20$ & $23.00^{c} \pm 0.00$ \\
\hline & LSD & 0.70 & 1.90 & 2.06 \\
\hline \multirow[t]{4}{*}{ D. Kwandawa } & 0 & $30.30^{\mathrm{a}} \pm 3.65$ & $23.00^{\mathrm{a}} \pm 2.00$ & $75.20^{\mathrm{a}} \pm 1.68$ \\
\hline & 30 & $25.67^{\mathrm{b}} \pm 7.30$ & $18.30^{\mathrm{b}} \pm 4.10$ & $67.10^{\mathrm{b}} \pm 3.70$ \\
\hline & 60 & $20.00^{c} \pm 3.00$ & $15.30^{\mathrm{c}} \pm 3.20$ & $53.80^{\mathrm{c}} \pm 3.90$ \\
\hline & LSD & 2.75 & 1.95 & 4.68 \\
\hline \multirow[t]{4}{*}{ Ganwon Falke } & 0 & $24.00^{\mathrm{a}} \pm 1.00$ & $17.67^{\mathrm{a}} \pm 1.50$ & $95.10^{\mathrm{a}} \pm 4.50$ \\
\hline & 30 & $17.30^{\mathrm{b}} \pm 2.50$ & $12.67^{\mathrm{b}} \pm 2.08$ & $89.40^{\mathrm{b}} \pm 2.90$ \\
\hline & 60 & $15.30^{\mathrm{b}} \pm 0.58$ & $8.30^{\mathrm{c}} \pm 2.00$ & $83.10^{\mathrm{b}} \pm 3.80$ \\
\hline & LSD & 2.39 & 1.86 & 4.95 \\
\hline \multirow[t]{4}{*}{ D. Dogarawa } & 0 & $28.30^{\mathrm{a}} \pm 306$ & $22.30^{\mathrm{a}} \pm 3.20$ & $136.03^{\mathrm{a}} \pm 32.20$ \\
\hline & 30 & $23.30^{\mathrm{b}} \pm 2.52$ & $15.67^{\mathrm{b}} \pm 1.10$ & $62.10^{\mathrm{b}} \pm 13.09$ \\
\hline & 60 & $16.30^{c} \pm 0.58$ & $11.00^{\mathrm{c}} \pm 1.00$ & $40.50^{c} \pm 9.30$ \\
\hline & LSD & 2.67 & 1.90 & 6.76 \\
\hline \multirow[t]{4}{*}{ Roma } & 0 & $19.67^{\mathrm{a}} \pm 5.00$ & $14.67^{\mathrm{a}} \pm 1.50$ & $84.60^{\mathrm{a}} \pm 5.00$ \\
\hline & 30 & $14.30^{\mathrm{b}} \pm 1.10$ & $11.30^{\mathrm{b}} \pm 1.15$ & $43.60^{\mathrm{b}} \pm 1.00$ \\
\hline & 60 & $9.67^{\mathrm{c}} \pm 4.90$ & $4.67^{\mathrm{c}} \pm 2.31$ & $41.10^{\mathrm{b}} \pm 1.88$ \\
\hline & LSD & 1.90 & 1.87 & 4.78 \\
\hline \multirow[t]{4}{*}{ UTC } & 0 & $16.67^{\mathrm{a}} \pm 1.10$ & $12.00^{\mathrm{a}} \pm 2.00$ & $104.30^{\mathrm{a}} \pm 4.01$ \\
\hline & 30 & $11.30^{\mathrm{b}} \pm 2.52$ & $7.30^{\mathrm{b}} \pm 1.53$ & $76.50^{\mathrm{b}} \pm 2.62$ \\
\hline & 60 & $7.30^{c} \pm 1.53$ & $3.67^{\mathrm{c}} \pm 1.10$ & $67.70^{c} \pm 1.15$ \\
\hline & LSD & 2.01 & 1.60 & 5.07 \\
\hline \multirow[t]{4}{*}{ Rio Grande } & 0 & $16.67^{\mathrm{a}} \pm 4.90$ & $11.30^{\mathrm{a}} \pm 3.79$ & $87.57^{\mathrm{a}} \pm 1.62$ \\
\hline & 30 & $13.00^{\mathrm{b}} \pm 1.00$ & $8.67^{\mathrm{b}} \pm 0.58$ & $75.40^{\mathrm{b}} \pm 1.50$ \\
\hline & 60 & $5.30^{c} \pm 2.00$ & $3.00^{\mathrm{c}} \pm 1.00$ & $68.57^{\mathrm{c}} \pm 0.81$ \\
\hline & LSD & 2.06 & 1.57 & 4.37 \\
\hline
\end{tabular}


Table 3 Continued: Effects of salt concentration on floral characters, number of fruits and fruits weight at harvest in different tomato cultivars studied.

\begin{tabular}{|c|c|c|c|c|}
\hline $\begin{array}{l}\text { Accessions/ } \\
\text { Landraces }\end{array}$ & $\begin{array}{l}\text { Treatment } \\
\text { (mgL-1) }\end{array}$ & Number of Flowers & Number of Fruit & Fruit Weight (g) \\
\hline \multirow[t]{4}{*}{ Giofranco F. } & 0 & $21.30^{\mathrm{a}} \pm 1.53$ & $12.67^{\mathrm{a}} \pm 2.08$ & $92.60^{\mathrm{a}} \pm 2.30$ \\
\hline & 30 & $15.67^{\mathrm{b}} \pm 2.89$ & $9.00^{\mathrm{b}} \pm 1.00$ & $79.00^{\mathrm{b}} \pm 0.52$ \\
\hline & 60 & $9.67^{\mathrm{c}} \pm 2.00$ & $5.67^{\mathrm{c}} \pm 2.00$ & $68.17^{\mathrm{c}} \pm 0.50$ \\
\hline & LSD & 2.60 & 1.87 & 4.75 \\
\hline \multirow{4}{*}{ UC82B } & 0 & $14.30^{\mathrm{a}} \pm 4.51$ & $10.00^{\mathrm{a}} \pm 2.00$ & $104.30^{\mathrm{a}} \pm 3.41$ \\
\hline & 30 & $11.00^{\mathrm{b}} \pm 2.00$ & $8.00^{\mathrm{a}} \pm 1.73$ & $88.87^{\mathrm{b}} \pm 1.140$ \\
\hline & 60 & $7.67^{\mathrm{c}} \pm 1.53$ & $5.00^{\mathrm{b}} \pm 1.00$ & $67.40^{c} \pm 7.09$ \\
\hline & LSD & 2.06 & 2.65 & 5.06 \\
\hline \multirow[t]{4}{*}{ Indian tomato } & 0 & $33.00^{\mathrm{a}} \pm 3.00$ & $28.00^{\mathrm{a}} \pm 2.65$ & $102.97^{\mathrm{a}} \pm 1.79$ \\
\hline & 30 & $25.67^{\mathrm{b}} \pm 1.50$ & $20.67^{\mathrm{b}} \pm 2.08$ & $96.07^{\mathrm{b}} \pm 2.35$ \\
\hline & 60 & $19.30^{c} \pm 0.58$ & $14.30^{\mathrm{c}} \pm 0.58$ & $88.50^{c} \pm 1.22$ \\
\hline & LSD & 3.07 & 2.07 & 6.09 \\
\hline \multirow[t]{4}{*}{ Tomato Peto } & 0 & $32.67^{\mathrm{a}} \pm 1.53$ & $26.67^{\mathrm{a}} \pm 1.10$ & $82.00^{\mathrm{a}} \pm 13.00$ \\
\hline & 30 & $30.30^{\mathrm{a}} \pm 0.58$ & $25.30^{\mathrm{a}} \pm 0.58$ & $69.40^{\mathrm{b}} \pm 0.60$ \\
\hline & 60 & $25.30^{\mathrm{b}} \pm 2.52$ & $20.00^{\mathrm{b}} \pm 1.00$ & $58.50^{\mathrm{c}} \pm 1.60$ \\
\hline & LSD & 3.18 & 2.01 & 4.90 \\
\hline \multirow[t]{4}{*}{ Tropimech } & 0 & $25.30^{\mathrm{a}} \pm 0.57$ & $18.67^{\mathrm{a}} \pm 3.79$ & $98.07^{\mathrm{a}} \pm 4.00$ \\
\hline & 30 & $21.30^{\mathrm{b}} \pm 150$ & $14.30^{\mathrm{b}} \pm 1.50$ & $78.77^{\mathrm{b}} \pm 4.62$ \\
\hline & 60 & $14.00^{\mathrm{c}} \pm 3.40$ & $8.00^{c} \pm 3.60$ & $67.70^{\mathrm{c}} \pm 1.10$ \\
\hline & LSD & 2.86 & 1.90 & 4.88 \\
\hline \multirow[t]{4}{*}{ Cherry } & 0 & $21.30^{\mathrm{a}} \pm 3.20$ & $14.30^{\mathrm{a}} \pm 1.53$ & $85.43^{\mathrm{a}} \pm 2.08$ \\
\hline & 30 & $16.30^{\mathrm{b}} \pm 2.00$ & $13.00^{\mathrm{a}} \pm 1.70$ & $66.60^{\mathrm{b}} \pm 7.20$ \\
\hline & 60 & $38.30^{\mathrm{c}} \pm 4.60$ & $6.67 \pm^{\mathrm{b}} 3.20$ & $60.17^{\mathrm{b}} \pm 7.48$ \\
\hline & LSD & 2.70 & 1.70 & 4.84 \\
\hline \multirow[t]{4}{*}{ Heirloom } & 0 & $24.30^{\mathrm{a}} \pm 2.89$ & $18.30^{\mathrm{a}} \pm 2.52$ & $89.37^{\mathrm{a}} \pm 2.30$ \\
\hline & 30 & $16.30^{\mathrm{b}} \pm 0.58$ & $12.00^{\mathrm{b}} \pm 1.00$ & $75.50^{\mathrm{b}} \pm 8.20$ \\
\hline & 60 & $15.30^{\mathrm{b}} \pm 2.31$ & $9.30^{c} \pm 2.08$ & $54.90^{\mathrm{c}} \pm 6.18$ \\
\hline & LSD & 2.76 & 1.85 & 4.89 \\
\hline
\end{tabular}

Values represent means and standard deviation of floral count, number of fruit and fruit weight at harvest. Mean in a column of a cultivar with the same superscript are not significantly different $(\mathrm{P}<0.05)$.

\section{Discussions}

In this study it was observed that salinity significantly decreased vegetative parameters with increase in salt concentration. In all the twenty accessions/landraces studied, control had the highest vegetative parameters (number of leaves, plant height, leaf area, root length and dry matter accumulation) followed by plants treated with $30 \mathrm{mgL}^{-1}$ of Salinity. The lowest vegetative parameters were observed on plants treated with $60 \mathrm{mgL}^{-1}$ of Salinity. Control had the highest number of leaves, plant height, root length, leaves area and dry matter accumulation. This is in accordance with the findings of Gumi et al. [6] on effect of growth biochemical parameters and ion homeostasis in Solanum lycopersicum. Root and shoot length are the most important parameters for salinity because of the direct contact of root with the soil and absorp water and shoot supply to the rest of plant body. For this reason, root and shoot length provides an important clue to the response of plant to salinity [12]. As salinity is first perceived in the root, it is likely that root derived signal, presumably abscsic acid is formed which directly or indirectly down regulates leaf expansion rate [14].

Decrease in number of flowers, number of fruits and fruit weight at harvest had been reported in tomato under salinity in a concentration dependent manner $[6,16]$. This is in accordance with the findings of the present study. The chemical potential of saline solution initially establish a water potential imbalance between the apoplast and symplast that lead to turgor decrease, which if severe enough can leads to growth reduction [2]. Similar observations have been reported in Capsicum annuum and Capsicum chinense by Ja'afar et al. [11], the report shows significant decrease in all the vegetative parameters, floral and fruit number in the two species with increasing salt concentration. Salinity induces proteomic changes in tomato [17], proteins and other macro 
molecules play a key role in flower formation and fruit quality. Amini et al. [1] reported highest floral count and number of fruit on tomato plant at control, with lowest number at plant treated with salt. Similar result was also reported by Reddy et al. [15] in Jatropha curcas and in cabbage, sugar beet, paniculate amaranth and pak-choi by Muhammad et al. [12].

\section{Conclusion}

From the findings of this study, controls recorded the highest number of leaves, plant height, root length, leaf area, number of flowers, and number of fruits, dry matter accumulation and weight of fruit at harvest. However, salinity also affected number of flowers and number of fruits.

\section{References}

[1] F. Amini et al., Protein Pattern changes in tomato under in vitro salinity, Russian Journal of Plant Physiology. 54 (2007) 464-471.

[2] H.J. Bohnert, R.G. Jensen, Strategies for engineering water stress tolerance in plants, Trend in Biotechnology. 14 (1996) 89-97.

[3] Central Bank of Nigeria, Consumer reports on crops. 12 (2012) 23-37.

[4] M.R. Foolad, Genome mapping and molecular breeding of tomato, International Journal of Plant Genomics. 2 (2007) 643-658.

[5] GAIN, Reort on assessment of commodity and trade issues, Gain and Feed Animals, 2018. Available: http/www.proshoreng.com. Retrieved: 11/02 2018.

[6] A.M. Gumi et al., Salinity Stress: Effects on growth, biochemical parameters and ion homeostasis in Solanum lycopersicum L. (Cv. Daneka), Central European Journal of Experimental Biology. 2(3) (2013) 20-25.

[7] M. Hasanuzzaman et al., Potential use of halophytes to remediate saline soils, Journal of Biomedical Research International. 45 (2014) 589-641.

[8] R. Hunt, Plant Growth Analysis, Oxford University Press, London, UK, 1981.

[9] IPGRI, Crop parameters. AquaCrop Annex 1 Version 4.0, 2005.

[10] IPGRI, Plant Genome Databases. SolGenes (for tomato) was developed by Cornell University with funding from the Plant Genome Project of the USDA, 2015. Available: http://probe.nalusda.gov:8300.

[11] U. Ja'afar, A.A. Aliero, A study on the vegetative and osmolyte accumulation of Capsicum frutescens L. under zinc stress, International Journal of Scientific Research and Engineering Studies. 2(8) (2015) 23-27.

[12] J. Muhammad et al., Studies on germination and growth of cabbage, sugar beet, paniculate and pak-choi, Journal of Central European Agriculture. 7 (2006) 273-281.

[13] R. Munns, R.A. James, A. Läuchli, Approaches to increasing the salt tolerance of wheat and other cereals, Journal of Experimental Botany. 57 (2006) 1025-1043.

[14] J.B. Passioura, Root signals control leaf expansion in wheat seedlings growing in drying soil, Australian Journal of Plant Physiology. 15 (1988) 687-693.

[15] M.P. Reddy et al., Effects of salinity on growth, ion accumulation, protein, proline content and antioxidant enzymes activity in callus culture of Jatropha curcas, Biologia. 63 (2008) 378-382.

[16] D.W. Rush, E. Epstein, Genotypic responses to salinity: differences between salt sensitive and salt-tolerant genotypes of the tomato, Journal of Plant Physiology. 57 (2006) 162-166. 
[17] R.A. Shibli et al., Physiological and biochemical responses of tomato micrshoots to induce salinity stress with associated ethylene accumulation, Plant Growth Regulation. 51 (2007) 159-169.

[18] J. Singh, E.V.D. Sastry, V. Singh, Effect of salinity on tomato (Lycopersicon esculentum Mill.) during seed germination stage, Journal of Plant Physiology and Molecular Biology. 18 (2012) 45-50.

[19] J.L. Van Hintum, A computer compatible system for scoring heterogeneous populations, Genetic Resources and Crop Evolution. 40 (1993) 133-136.

[20] T. Wada et al., Effect of foliar application of calcium solutions on the incidence of blossomend rot of tomato fruit, Journal of the Japanese Society for Horticultural Science. 65 (1996) $553-558$.

[21] T. Wallace, Rural Development through Irrigation: Studies in town on the Kano River Project, CSER, ABU, Zaria, Nigeria, 2013.

[22] Y. Zhai, Q. Yang, M. Hou, The Effects of Saline Water Drip Irrigation on Tomato Yield, Quality and Blossom-end rot incidence - A Case Study in the South of China, PLoS ONE. 10 (2015) e 0142204.

[23] X. Zhang et al., Recent progress in drought and salt tolerance studies in Brassica crops, Journal of Science Breeding. 64 (2014) 60-73. 